\title{
Identification of potentially relevant genes for myocardial infarction using RNA sequencing data analysis
}

\author{
QIANG ZHAO ${ }^{1 *}, \mathrm{KE} \mathrm{WU}^{2 *}$, NANNAN $\mathrm{LI}^{3}, \mathrm{ZHENGMEI} \mathrm{LI}^{4}$ and FENGLIN JIN ${ }^{1}$ \\ ${ }^{1}$ Department of Cardiology, The Affiliated Hospital of Taishan Medical University of \\ Shandong Province; Departments of ${ }^{2}$ Cardiology and ${ }^{3}$ Respiration Medicine, \\ Central Hospital of Tai'an of Shandong Province; ${ }^{4}$ Department of Radiology, Taishan Medical \\ University of Shandong Province, Tai'an, Shandong 271000, P.R. China
}

Received May 2, 2017; Accepted October 6, 2017

DOI: $10.3892 / e t m .2017 .5580$

\begin{abstract}
Myocardial infarction (MI) is a heart disease with high morbidity and mortality rates, thus it is critical to identify genes that serve roles during its pathogenesis. The objective of the present study was to identify potentially relevant genes during the progression of the disease. Blood samples from patients with $\mathrm{MI}$ and normal controls ( $\mathrm{n}=3 /$ group) were obtained, the RNA was extracted and cDNA libraries were established. RNA sequencing (RNA-seq) was performed on a HiSeq 2500 platform and fragments per kilobase of exon per million fragments mapped was utilized to calculate the gene expression value following preprocessing of the RNA-seq data. Electronic validation of several identified differentially expressed genes (DEGs) was performed on a Gene Expression Omnibus (GEO) dataset GSE59867 (390 cases and 46 healthy controls). Functional enrichment and protein-protein interaction network analysis was conducted for DEGs. A total of 977 DEGs, including 817 upregulated and 160 downregulated genes were identified in patients with MI. These DEGs were significantly enriched for 'positive regulation of the immune system process,' 'inflammatory response,' 'regulation of I-kappaB-kinase/NF-kappaB signaling' and 'TNF signaling pathway'. A protein-protein interaction network of the top 40 DEGs was used to identify high degree genes, including interferon induced protein with tetratricopeptide repeats 3 (IFIT3), MX dynamin like GTPase 1 (MX1), major histocompatibility complex, class II, DQ $\alpha 1$ (HLA-DQA1), RAR related orphan receptor A (RORA), prostaglandin D2 synthase (PTGDS), cysteine rich protein 2 (CRIP2), collagen type VI $\alpha 2$ chain (COL6A2) and S100 calcium binding
\end{abstract}

Correspondence to: Dr Fenglin Jin, Department of Cardiology, The Affiliated Hospital of Taishan Medical University of Shandong Province, 706 Taishan Street, Tai'an, Shandong 271000, P.R. China E-mail: jinfenglin1234@163.com

*Contributed equally

Key words: myocardial infarction, RNA sequencing, differentially expressed genes, protein-protein interaction protein $\mathrm{P}$ (S100P). The results of validation in the GEO dataset were consistent with the sequencing analysis. A total of eight genes, including IFIT3, MX1, HLA-DQA1, RORA, PTGDS, CRIP2, COL6A2 and S100P may therefore be considered as potentially relevant genes in the pathology of MI.

\section{Introduction}

Plaques form in the coronary arteries during atherosclerosis, which impede blood flow. Rupturing of plaques leads to the exposure of arterial blood to pro-thrombotic substances (1). If the intravascular thrombus completely blocks the artery, this causes myocardial infarction (MI), a type of heart disease with a high morbidity rate and mortality rate of 5\% (1). Under this circumstance, the left ventricle undergoes a number of changes in shape, size and function, which may lead to heart failure (2). There are multiple factors associated with MI, including sex, age, smoking and complications, including hypertension and diabetes mellitus (3). In primary care, the diagnosis of MI is recorded using Read codes (4) and previous studies have reported lists of Read codes that may be used to identify acute MI $(5,6)$. Clinically, antithrombotic therapy or revascularization is the primary treatment method of type $1 \mathrm{MI}$, which is defined as myocardial ischemia caused by primary coronary events such as plaque rupture (7). Percutaneous coronary intervention in a timely manner is the primary treatment for acute ST segment elevation MI (7). In addition, various cardiac medications, including aspirin, $\beta$-blockers, lipid lowering agents and angiotensin converting enzyme inhibitors have been used in patients with acute MI $(8,9)$.

Although some targeted treatments, including $\beta$-blockers and angiotensin converting enzyme inhibitors, have made considerable progress in treating heart failure in patients post-MI (10), further understanding of the potential pathological mechanism of MI is required. An improved understanding of the underlying genes and molecular mechanisms, which increase the risk of MI, is essential to improve its diagnosis, prevention and treatment. Previous reports have indicated some of the potential risk genes and micro-RNAs (miRNAs) in MI. Gao et al (11) suggested that C-C motif chemokine ligand 5, B-cell CLL/lymphoma 3, nuclear receptor subfamily 2 group $\mathrm{C}$ member 2, MYC associated factor X, general transcription 
factor IIIC subunit 2 and nuclear receptor coactivator 7 serve an important role in the development of acute MI. Several higher expression genes, including F-box and leucine rich repeat protein 4, enoyl-CoA hydratase domain containing 3, potassium voltage-gated channel subfamily E regulatory subunit 1, TAO kinase 2, aurora kinase B, ERG and FK506 binding protein 5 and lower expression genes, including myocardial infarction associated transcript, poliovirus receptor-related 3 and PZP were also demonstrated to be present in ST-segment elevation MI compared to non-ST-segment elevation MI (12). In addition, it is reported that elevated cardiac-specific miR-208a in plasma may be a potential biomarker for the early detection of MI (13).

RNA sequencing (RNA-seq) is a progressive method for transcription profiling that provides more precise information about gene expression (14). In the present study, RNA-seq was used to evaluate the gene expression between blood samples from patients with MI and a control group of healthy patients, and a large number of differentially expressed genes (DEGs) were identified. The biological processes, signaling pathways and protein-protein interactions (PPIs) of the identified DEGs were studied. The results were used to further understand the underlying molecular mechanisms of and the potentially relevant genes in the development of MI.

\section{Materials and methods}

Collection of patient samples. In the current study, patients with MI were enrolled according to the following conditions: i) Clinical history of ischemic chest pain; ii) dynamic evolution of electrocardiogram and iii) concentration change of myocardial necrosis related serum biomarkers. All patients with MI had no serious infections, endocrine disease, liver and kidney disease, severe neurological disease or blood system disease. Those patients with angina, acute pericarditis, acute pulmonary embolism, aortic dissection, myocarditis, cardiomyopathy, coronary artery malformations and multiple arteritis were excluded. For the normal control group, there was no statistical difference in age, gender, high density lipoprotein and low density lipoprotein compared with the MI group. A total of 3 patients with MI and 3 healthy control were enrolled between December 2015 and February 2016 in the Central Hospital of Tai'an of Shandong Province (Shandong, China). The MI group consisted of one female and two males ages between 46-59 years and the control group consisted of one female and two males aged between $45-56$ years. Blood samples $(10 \mathrm{ml})$ were obtained from all patients and then stored in $-80^{\circ} \mathrm{C}$ freezer for subsequent RNA extraction. The clinical results of patients with $\mathrm{MI}$ and normal controls are presented in Table I. The current study was approved by the Ethics Committee of Central Hospital of Tai'an of Shandong Province and written informed consent was obtained from all patients.

Patient sample collection, RNA isolation and sequencing. Total RNA were extracted, according to the manufacturer's protocol, using TRIzol reagent (Invitrogen; Thermo Fisher Scientific, Inc., Waltham, MA, USA) and an RNeasy mini kit (Qiagen AB, Sollentuna, Sweden), respectively. The quality and quantity of RNA was determined on a Nanodrop
ND-2000 spectrophotometer (Thermo Fisher Scientific, Inc., Pittsburgh, PA, USA). 1.5\% agarose gel electrophoresis was used to further verify RNA integrity.

The mRNA was purified from total RNA with a TruSeq RNA library preparation kit (Illumina, Inc., San Diego, CA, USA) and fragmented into sizes of 200 bp using fragmentation buffer (Illumina, Inc.). The fragmented RNA was converted into double-stranded cDNA in a mixture consisting of reverse transcriptase, buffer, dNTPs and primers (Illumina, Inc.) for $5 \mathrm{~min}$ at $65^{\circ} \mathrm{C}$ followed by $60 \mathrm{~min}$ at $42^{\circ} \mathrm{C}$ and $5 \mathrm{~min}$ at $72^{\circ} \mathrm{C}$. Following end repair, adapter ligation and gel purification, the 200 bp cDNA was isolated into 300-nt fragments. A QIAquick PCR kit (Qiagen AB) was used to estimate the relative concentration of the library using a Bioanalyzer 2100 (Agilent Technologies, Inc., Santa Clara, CA, USA) according to the manufacturer's protocol. A HiSeq 2500 platform (Illumina, Inc.) was then applied to perform sequencing. SeqPrep 1.3.2-1 (github.com/jstjohn/SeqPrep) and Sickle V1.0 (github.com/najoshi/sickle) software were used to remove low quality sequences, including ambiguous nucleotides and adaptor sequences of the raw sequencing data. The cleaned sequencing reads were aligned to the University of California Santa Cruz (UCSC, http://genome.ucsc.edu) human reference genome (build hg19) through TopHat 1.3.1 (http://ccb. jhu.edu/software/tophat/index.shtml). The comparison of the reference genome reads with different samples is presented in Table II.

Identification of DEGs. The fragment assembly and relative expression of the reads with the normalized RNA-Sequencing fragment counts were performed using Cufflinks software 2.2.1 (http://cufflinks.cbcb.umd.edu/). Fragments per kilobase of exon per million fragments mapped was used to determine the transcription abundance of genes. A paired t-test was performed for statistical analysis. A false discovery rate of $<0.05$ was determined to indicate statistically significant differences.

Electronic validation of DEGs in the Gene Expression Omnibus dataset. The Gene Expression Omnibus (GEO, http://www.ncbi.nlm.nih.gov/geo) database was used to validate the expression of the identified DEGs. In the current study, the mRNA profile of $390 \mathrm{MI}$ cases and 46 normal controls was obtained from the GSE59867 dataset. The expression level of the selected targeted DEGs between MI cases and normal controls were compared and the difference of expression level was displayed using box-plots.

Functional enrichment analysis of DEGs. Gene Ontology (GO, geneontology.org/) enrichment analysis and Kyoto Encyclopedia of Genes and Genomes (KEGG, genome. $\mathrm{jp} / \mathrm{kegg} /$ pathway.html) pathway enrichment analysis was performed to select DEGs using the Database for Annotation, Visualization and Integration Discovery (DAVID 6.8, david. abcc.Ncifcrf.gov/) online software. The cut-off value for significance was $\mathrm{P}<0.05$.

Construction of PPI network. BioGrid database (thebiogrid. org) was used to retrieve the predicted interactions between the top 40 DEGs (20 upregulated and 20 downregulated) and 
Table I. The clinical information of patients with MI and normal controls.

\begin{tabular}{|c|c|c|c|c|c|c|c|c|}
\hline & Sample & Age & Sex & $\begin{array}{l}\text { Height } \\
\text { (m) }\end{array}$ & $\begin{array}{l}\text { Weight } \\
(\mathrm{kg})\end{array}$ & BMI & $\begin{array}{l}\text { Hypertension } \\
\text { history }\end{array}$ & $\begin{array}{c}\text { Diabetes } \\
\text { history }\end{array}$ \\
\hline MI & Peripheral Blood & 50 & Female & 1.6 & 68 & 26.5 & No & No \\
\hline MI & Peripheral Blood & 46 & Male & 1.67 & 75 & 26.9 & Yes & No \\
\hline MI & Peripheral Blood & 59 & Male & 1.71 & 68 & 23.4 & No & No \\
\hline Normal control & Peripheral Blood & 45 & Female & 1.63 & 70 & 26.3 & No & No \\
\hline Normal control & Peripheral Blood & 49 & Male & 1.65 & 70 & 25.7 & Yes & No \\
\hline Normal control & Peripheral Blood & 56 & Male & 1.72 & 71 & 23.9 & No & No \\
\hline
\end{tabular}

BMI, body mass index.

proteins. Interplayed pairs, which have a confidence score $>0.4$, were selected to build the PPI network and were visualized using Cytoscape 3.3.0 (cytoscape.org/) software. A node in the PPI network denotes DEGs and the degree of a node represents the number of proteins that are involved in the node.

\section{Results}

DEGs associated with MI. A total of 977 DEGs, including 817 upregulated and 160 downregulated genes, were identified in samples from patients with MI. The top 10 upregulated DEGs were histone cluster 1 H2B family member O, cluster of differentiation 177, mast cell expressed membrane protein 1 , LOC105376505, vanin 1, annexin A3, disheveled associated activator of morphogenesis 2, LOC105372578, interferon induced protein with tetratricopeptide repeats (IFIT) 1 and butyrophilin like 8 . The top 10 downregulated DEGs were major histocompatibility complex, class II, DR $\beta 5$, major histocompatibility complex, class II, DQ $\alpha 1$ (HLA-DQA1), major histocompatibility complex, class II, DR $\beta 1$, collagen type VI $\alpha 2$ chain (COL6A2), cysteine rich protein 2 (CRIP2), family with sequence similarity 153 member $\mathrm{A}$, tandem $\mathrm{C} 2$ domains, nuclear, protein kinase, Y-linked, pseudogene, SH3 and SYLF domain containing 1 and neural EGFL like 2. A heat map of the top 100 DEGs (50 upregulated and 50 downregulated) is presented in Fig. 1. Additionally, the electronic validation of eight DEGs in the Gene Expression Omnibus dataset (GSE59867) is presented in Fig. 2. MX1 was significantly upregulated in the MI group compared with control $(\mathrm{P}<0.05)$, while RORA was significantly downregulated in MI compared with control $(\mathrm{P}<0.001)$. IFIT3 $(\mathrm{P}>0.05)$ and S100P $(\mathrm{P}>0.05)$ were upregulated and PTGDS and HLA-DQA1 were downregulated in the MI compared with the control however, this difference was not significant. The expression trend of these genes was consistent with the RNA sequencing analysis. However, the expression of COL6A2 $(\mathrm{P}<0.01)$ and CRIP2 ( $\mathrm{P}>0.05)$ were upregulated in the MI group compared with control, which was not in line with the RNA sequencing analysis.

Enrichment analysis of DEGs. GO enrichment analysis and KEGG pathway analysis demonstrated that the identified DEGs are significantly involved in the 'positive regulation of the immune system process,' 'inflammatory response,' 'adaptive immune response,' 'regulation of I-kappaB/NF-kappaB signaling,' 'hematopoietic cell lineage' and 'TNF signaling pathway'. Figs. 3 and 4 indicate the results of GO enrichment analysis and KEGG pathway analysis, respectively. The KEGG map of the nuclear factor (NF)- $\mathrm{\kappa B}$ and tumor necrosis factor (TNF) signaling pathways are presented in Figs. 5 and 6 , respectively.

PPI network of DEGs. Following the integration of protein information in the BioGrid database with the top 40 DEGs (20 upregulated and 20 downregulated), a PPI network of the identified DEGs was established. The primary nodes with high degrees $(\geq 10)$ were identified as follows: IFIT3 (degree, 66 ); MX dynamin like GTPase 1 (MX1; degree, 34); HLA-DQA1 (degree, 28); RAR related orphan receptor A (RORA; degree, 23); prostaglandin D2 synthase (PTGDS; degree, 15); CRIP2 (degree, 15); COL6A2 (degree, 12); and S100 calcium binding protein P (S100P; degree, 10) (Fig. 7).

\section{Discussion}

MI is a major cause of mortality worldwide and affects patient health and quality of life (1). In the current study, RNA-seq technology was applied to identify DEGs to investigate the roles served by important genes in MI. A total of 977 DEGs, with 817 upregulated genes and 160 downregulated genes, were identified. Following GO annotation and KEGG pathway analysis, it was determined that all of the DEGs were enriched with the 'positive regulation of the immune system process,' 'inflammatory response,' 'adaptive immune response,' 'regulation of I-kappaB/NF-kappaB signaling,' 'hematopoietic cell lineage' and 'TNF signaling pathway'. PPI analysis also identified several high-degree DEGs, suggesting that they serve an important role in MI.

There are a small number of immune cells in the adult mammalian heart and these immune cells expand in size following MI (15). Necrotic cardiomyocytes release danger signals, activating immune cells, which clear the matrix debris and dead cells, allowing the activation of cardiac repair (15). The extent of the inflammatory response may be used to identify the patient's outcomes, including heart function healing, acute myocardial rupture or dilatation (16). Post-MI inflammatory responses serve a role in cardiac repair and determine 


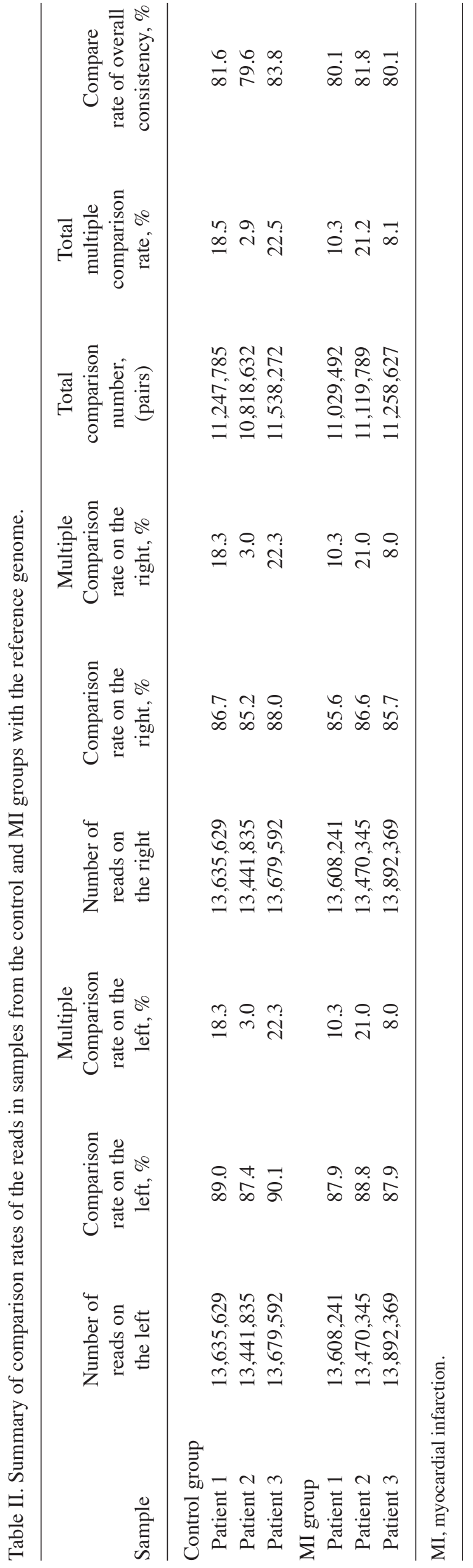

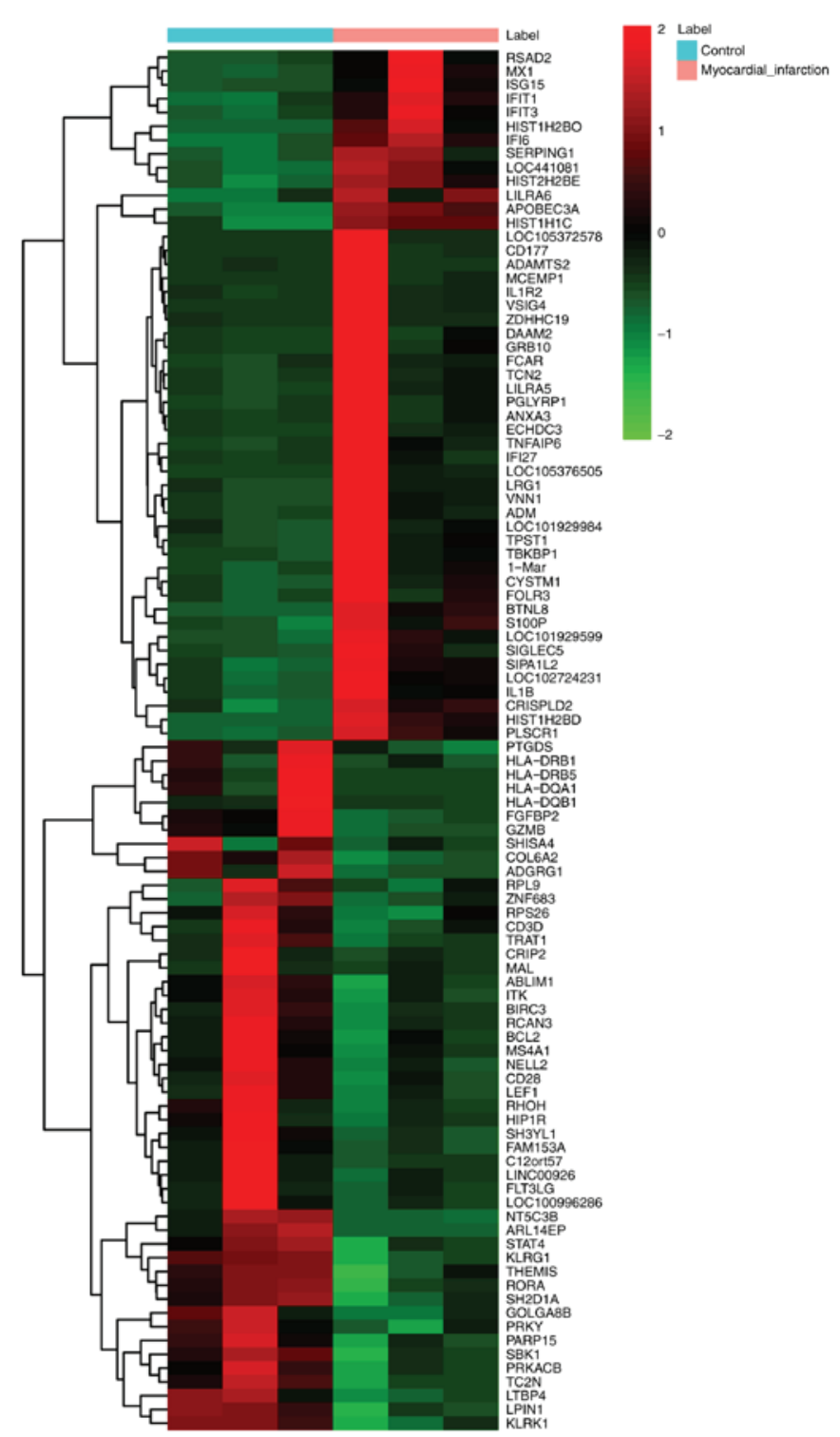

Figure 1. Heat map of clustering analysis of top 100 differentially expressed genes in myocardial infarction obtained using heatmap.2 (http://127.0.0.1:28428/library/gplots/html/heatmap.2.html).

the infarct size and quality of repair (17). In the current study, DEGs were significantly enriched in the biological processes of GO annotation, which further demonstrated the role they serve in the immune system and inflammatory response during MI.

NF- $\mathrm{KB}$ activation serves a critical role in coronary microembolism-induced cardiac dysfunction and advanced heart failure (18). For example, NF- $\mathrm{kB}$ activation and the subsequent inflammatory response contribute to cardiac dysfunction and maladaptive left ventricular remodeling following MI (19). The TNF signaling pathway protects the heart by ischemic preconditioning; however, this protective effect depends on the concentration of TNF- $\alpha(20,21)$. Therefore, the appropriate concentration of TNF- $\alpha$ is a critical factor in the determination of the outcome of cardiac injury, particularly following acute MI (22). In the present study, the DEGs were also involved in the NF- $\mathrm{kB}$ and TNF signaling pathways, thus, these genes may serve roles via these pathways in the development of cardiac dysfunction in MI. 

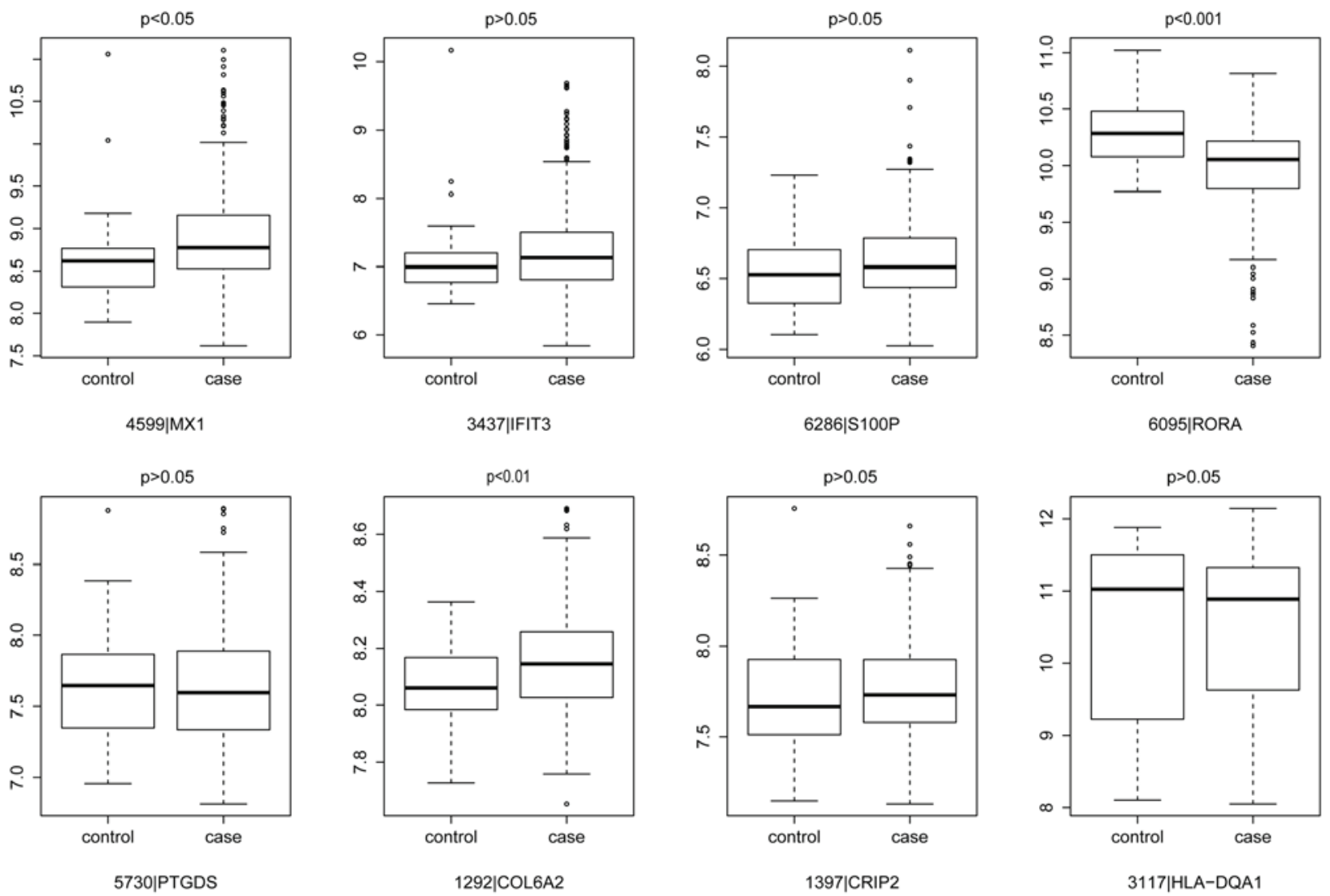

Figure 2. Electronic validation of eight differentially expressed genes in the Gene Expression Omnibus dataset (GSE59867). Electronic validation of MX1, IFIT3, S100P, RORA, PTGDS, COL6A2, CRIP2 and HLA-DQA1 in the control and case groups. MX1, MX dynamin like GTPase 1; IFIT3, interferon induced protein with tetratricopeptide repeats 3; S100P, S100 calcium binding protein P; RORA, RAR related orphan receptor A; PTGDS, prostaglandin D2 synthase; COL6A2, collagen type VI alpha 2 chain; CRIP2, cysteine rich protein 2; HLA-DQA1, major histocompatibility complex, class II, DQ $\alpha 1$.

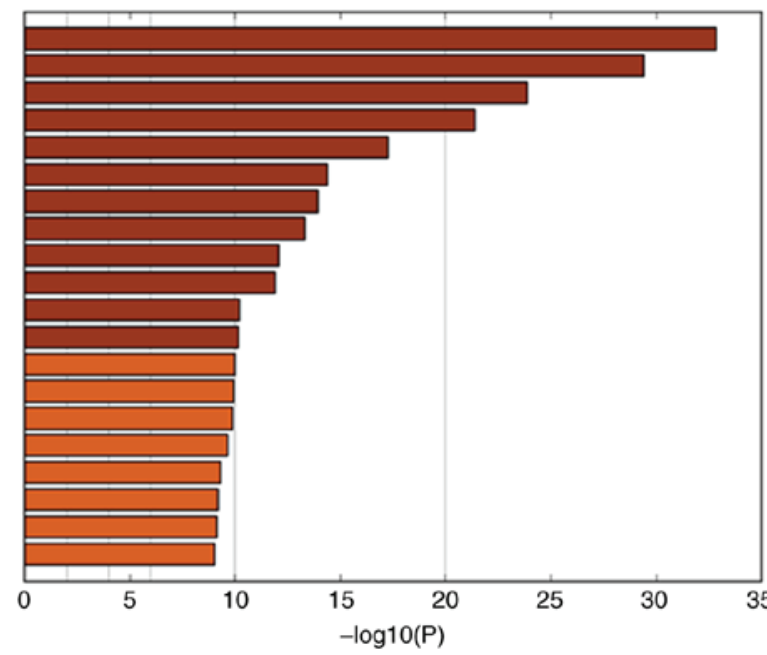

GO:0002684: positive regulation of immune system process GO:0001775: cell activation

GO:0006954: inflammatory response

GO:0034097: response to cytokine

GO:0002250: adaptive immune response

GO:0002274: myeloid leukocyte activation

GO:0002366: leukocyte activation involved in immune response

GO:0009617: response to bacterium

GO:0032940: secretion by cell

GO:0005773: vacuole

GO:0043069: negative regulation of programmed cell death GO:0001818: negative regulation of cytokine production

GO:1903706: regulation of hemopoiesis

GO:0002221: pattern recognition receptor signaling pathway

GO:0002697: regulation of immune effector process

GO:0010942: positive regulation of cell death

GO:0043122: regulation of I-kappaB kinase/NF-kappaB signaling

GO:1903901: negative regulation of viral life cycle

GO:0030099: myeloid cell differentiation

GO:0032609: interferon-gamma production

Figure 3. GO enrichment analysis of differentially expressed genes in myocardial infarction. GO, Gene Ontology.

To further study the function of the identified DEGs in MI, a PPI network was constructed. In the PPI network, several hub nodes were identified, which suggested that the hub genes may serve crucial roles in the pathology of MI. IFIT3 is a gene associated with innate immunity (23). The expression of IFIT3 is increased in transgenic mouse myocytes (24) and is upregulated in animal models of pathological cardiac hypertrophy (25). In the current study, the expression of IFIT3 was upregulated in patients with MI, which suggests it may function as an immunity response gene in MI.

Chen et al (10) indicated that MX1 was associated with MI. In the present study, MX1 was upregulated in MI, which suggested that it may be involved in the pathology of the disease. HLA-DQA1 is a member of the MHC Class II family and is a downregulated gene in patients with cardiorenal syndrome (26). In the present study, the decreased expression 


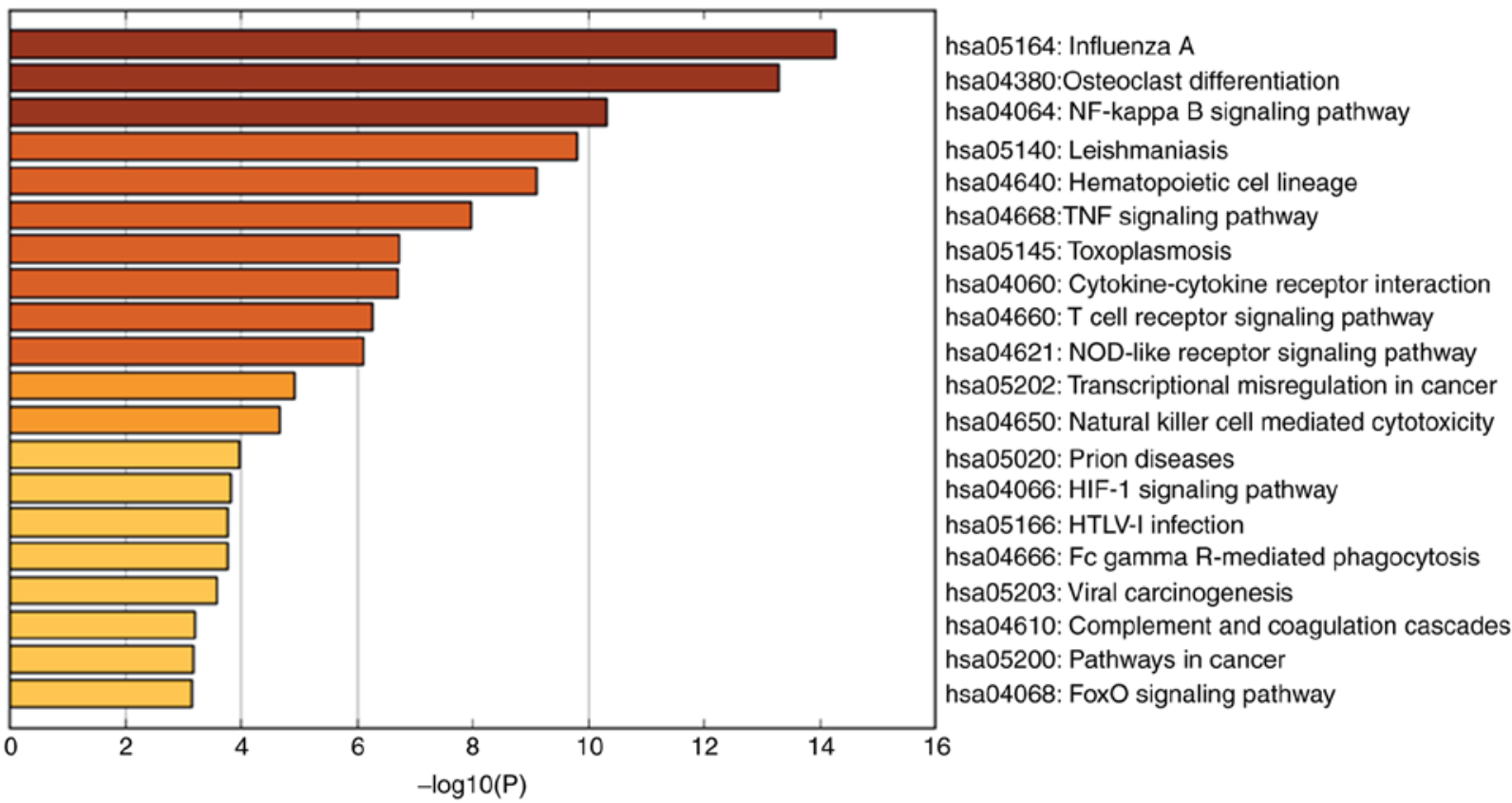

Figure 4. Kyoto Encyclopedia of Genes and Genomes pathway analysis of differentially expressed genes in myocardial infarction. NF, nuclear factor; TNF, tumor necrosis factor; HIF-1, hypoxia-inducible factor 1; HTLV-1, human T-lymphotropic virus-1.

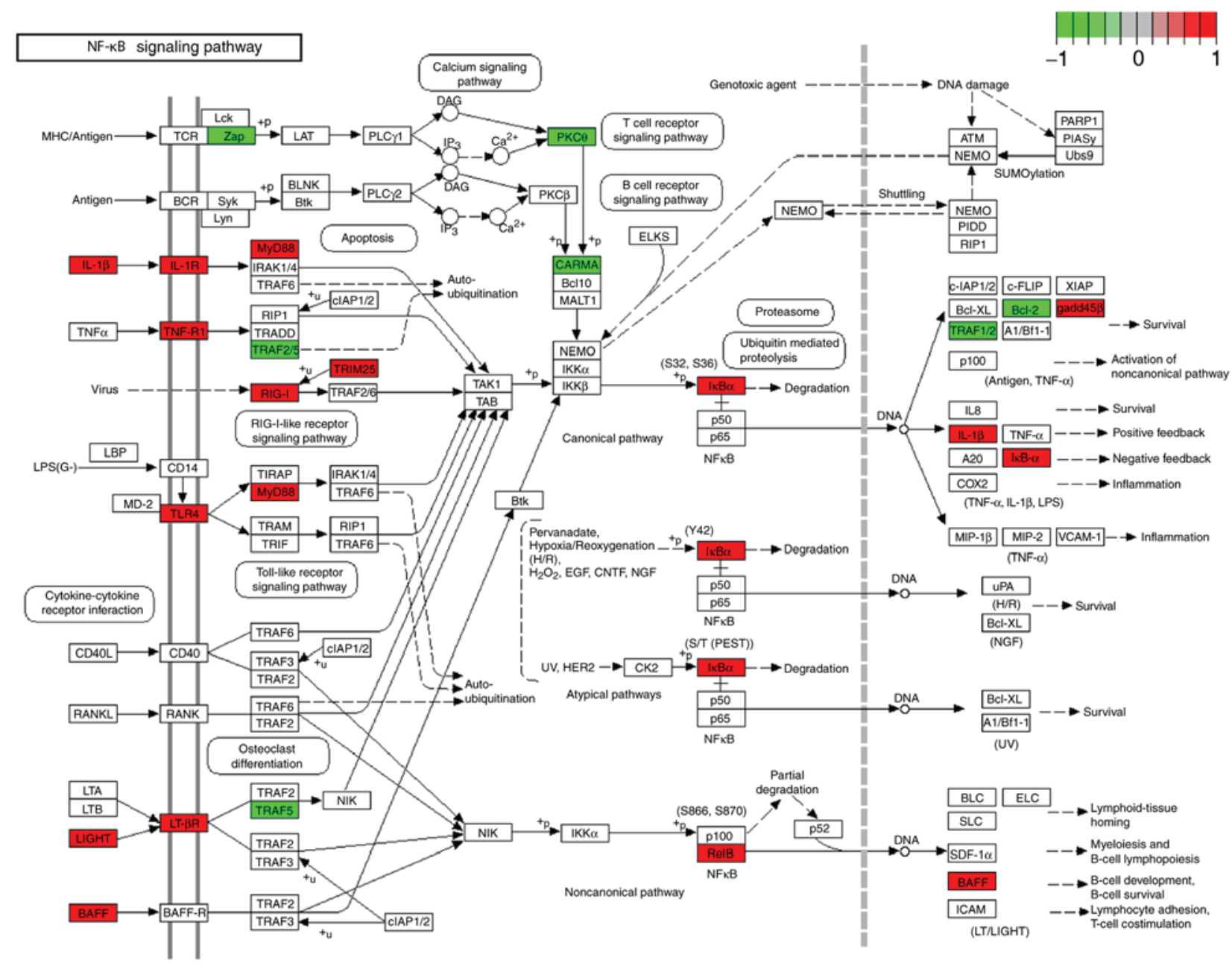

Data on KEGG graph

Figure 5. DEGs enriched KEGG map of the NF-кB signaling pathway in myocardial infarction. Colored rectangle represents the DEG the red and green

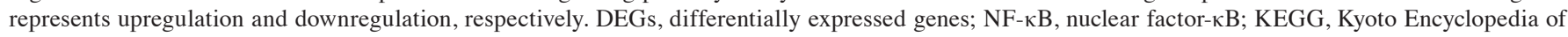
Genes and Genomes. 
TNF Signaling pathway
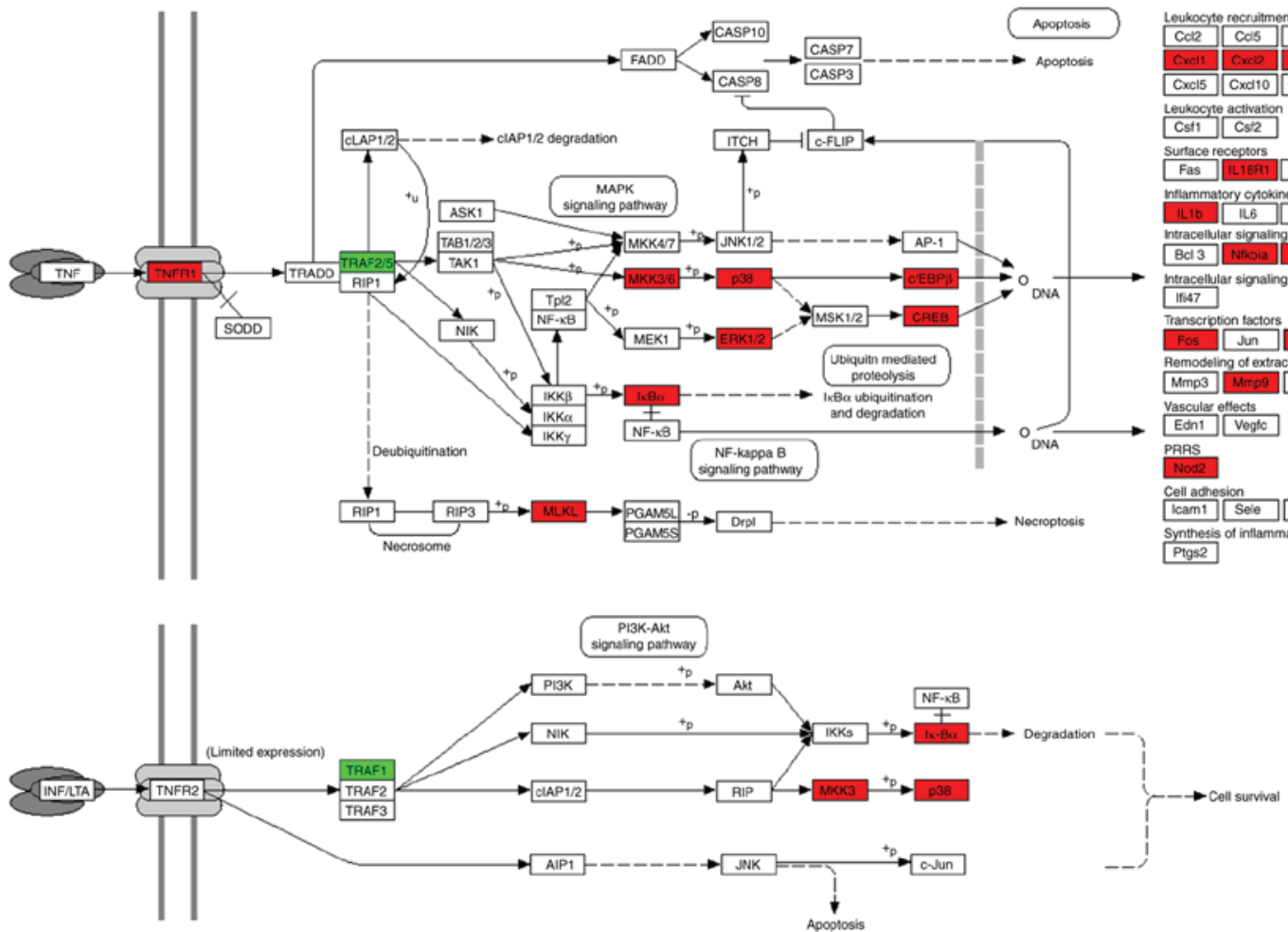

Data on KEGG graph

Figure 6. DEGs enriched KEGG map of the TNF signaling pathway in myocardial infarction. Colored rectangle represents the DEG, the red and green represents upregulation and downregulation, respectively. DEGs, differentially expressed genes; TNF, tumor necrosis factor; KEGG, Kyoto Encyclopedia of Genes and Genomes.

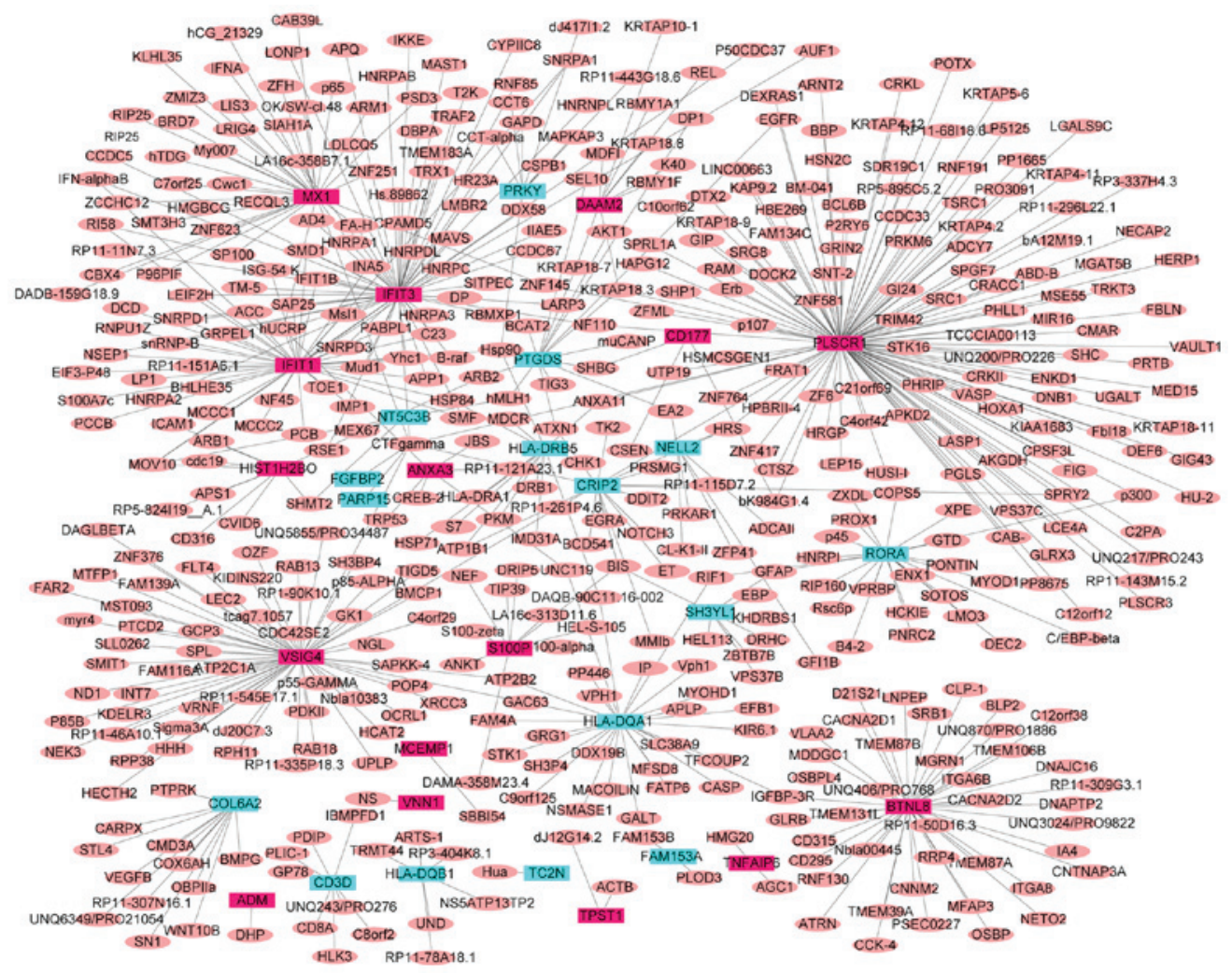

Figure 7. Protein-protein interaction network of the top 40 DEGs in myocardial infarction. Rectangle and ellipse represents the DEG and protein, respectively. Red represents an upregulated gene and blue represents a downregulated gene. DEGs, differentially expressed genes. 
of HLA-DQA1 in the blood of patients with MI was demonstrated. This suggested that HLA-DQA1 may serve a notable role in the pathology of MI.

RORA is a downstream effector molecule of circadian locomotor output cycles kaput genes $(27,28)$. Results of genome-wide association studies (GWAS) or GWAS meta-analyses have demonstrated that RORA is one of the primary risk factors for cardiovascular metabolism (29). Additionally, RORA serves a role in the regulation of the inflammatory response (30). In the current study, the expression of RORA was downregulated, which suggested that RORA may be associated with MI.

PTGDS is involved in the biosynthesis of prostaglandin D2 and promotes cardiomyocyte survival $(31,32)$. It serves a role in numerous diseases, including inflammatory, infectious and cardiovascular disease (33). Furthermore, PTGDS protein has been designated as a circulating marker in coronary artery disease $(34,35)$. In the present study, the expression of PTGDS was decreased, which indicated that it may be a biomarker of MI.

CRIP2 is a cysteine-rich protein, which is expressed in the heart endothelium during development and also in the adult heart $(36,37)$. CRIP2 is considered as a heart vascular marker (38). In the present study, CRIP2 expression was decreased in MI. However, the electronic validation result was not consistent with the sequencing analysis. This may be due to the size of the dataset used in the current study, thus larger datasets should perhaps be used for further investigations.

Cardiomyocyte death due to long-term ischemia induces infarction and robust adhesion molecule upregulation (39). In addition, COL6A2 is a cardiac fibroblast-enriched gene and encodes adhesion proteins $(40,41)$. In the current study, the expression of COL6A2 was decreased in patients with $\mathrm{MI}$, which indicated that COL6A2 may be involved in its development. However, it was upregulated in the results of electronic validation. This again may be due to the size of the dataset used in the current study and larger datasets should perhaps be used for further investigations.

Research has also demonstrated that S100P is associated with myocardial injury in patients and rat models of MI (42). The expression of S100P was increased in the serum of patients with acute coronary syndrome and was associated with infarct size (42). Similarly, the expression of S100P was increased in the current study, which further demonstrated its role in $\mathrm{MI}$.

In conclusion, several potentially relevant genes, including IFIT3, MX1, HLA-DQA1, RORA, PTGDS, CRIP2, COL6A2 and S100P, in MI were identified in the current study and these may serve important roles in the development of MI. However, there are certain limitations to the present study. The number of blood samples used for RNA-seq was small and a larger sample size is required. This is a preliminary study investigating MI using bioinformatics analysis. In vitro gene expression validation (such as real-time quantitative polymerase chain reaction), animal models (including gene overexpression and gene knockdown), and epigenetics studies (such as DNA methylation) are required to further investigate the function of identified genes in MI. However, the current study provides a basis for increasing the understanding of the gene expression profile in MI.

\section{References}

1. Wołkow PP, Drabik L, Totoń-Żurańska J, Kuś K, Foryś J, Słowik A, Pera J, Godlewski J, Tomala M, Żmudka K, et al: Polymorphism in the chemokine receptor 7 gene (CCR7) is associated with previous myocardial infarction in patients undergoing elective coronary angiography. Int J Immunogenet 43: 218-225, 2016.

2. Gajarsa JJ and Kloner RA: Left ventricular remodeling in the post-infarction heart: A review of cellular, molecular mechanisms and therapeutic modalities. Heart Fail Rev 16: 13-21, 2011.

3. Mahon NG, O'Rorke C, Codd MB, McCann HA, McGarry K and Sugrue DD: Hospital mortality of acute myocardial infarction in the thrombolytic era. Heart 81: 478-482, 1999.

4. Appraisal PC: NHS Connecting for Health. Betascript Publishing, 2005.

5. Bhattarai N, Charlton J, Rudisill C and Gulliford MC: Coding, recording and incidence of different forms of coronary heart disease in primary care. PLoS One 7: e29776, 2012.

6. Delaney JA, Daskalopoulou SS, Brophy JM, Steele RJ, Opatrny L and Suissa S: Lifestyle variables and the risk of myocardial infarction in the general practice research database. BMC Cardiovasc Disord 7: 38, 2007.

7. Reddy K, Khaliq A and Henning RJ: Recent advances in the diagnosis and treatment of acute myocardial infarction. World $\mathbf{J}$ Cardiol 7: 243-276, 2015.

8. Rosamond W, Flegal K, Friday G, Furie K, Go A, Greenlund K, Haase N, Ho M, Howard V, Kissela B, et al: Heart disease and stroke statistics-2007 update: A report from the american heart association statistics committee and stroke statistics subcommittee. Circulation 115: e69-e171, 2007.

9. Yusuf S, Sleight P, Held P and McMahon S: Routine medical management of acute myocardial infarction. Lessons from overviews of recent randomized controlled trials. Circulation 82: II117-II134, 1990.

10. Chen YF, Pottala JV, Weltman NY, Ge X, Savinova OV and Gerdes AM: Regulation of gene expression with thyroid hormone in rats with myocardial infarction. PLoS One 7: e40161, 2012.

11. Gao Y, Qi GX, Guo L and Sun YX: Bioinformatics analyses of differentially expressed genes associated with acute myocardial infarction. Cardiovasc Ther 34: 67-75, 2016.

12. Eicher JD, Wakabayashi Y, Vitseva O, Esa N, Yang Y, Zhu J, Freedman JE, McManus DD and Johnson AD: Characterization of the platelet transcriptome by RNA sequencing in patients with acute myocardial infarction. Platelets 27: 230-239, 2016.

13. Wang GK, Zhu JQ, Zhang JT, Li Q, Li Y, He J, Qin YW and Jing Q: Circulating microRNA: A novel potential biomarker for early diagnosis of acute myocardial infarction in humans. Eur Heart J 31: 659-666, 2010.

14. Ozsolak F and Milos PM: RNA sequencing: Advances, challenges and opportunities. Nat Rev Genet 12: 87-98, 2011.

15. Chen B and Frangogiannis NG: Immune cells in repair of the infarcted myocardium. Microcirculation, 2017 (In press).

16. Nian M, Lee P, Khaper N and Liu P: Inflammatory cytokines and postmyocardial infarction remodeling. Circ Res 94: 1543-1553, 2004.

17. Frangogiannis NG: Inflammation in cardiac injury, repair and regeneration. Curr Opin Cardiol 30: 240-245, 2015.

18. Su Q, Li L, Wang J, Zhou Y and Liu Y: Mechanism of programmed cell death factor $4 /$ nuclear factor- $\mathrm{\kappa B}$ signaling pathway in porcine coronary micro-embolization-induced cardiac dysfunction. Exp Biol Med (Maywood) 240: 1426-1433, 2015.

19. Onai Y, Suzuki J, Maejima Y, Haraguchi G, Muto S, Itai A and Isobe M: Inhibition of NF-\{kappa\}B improves left ventricular remodeling and cardiac dysfunction after myocardial infarction. Am J Physiol Heart Circ Physiol 292: H530-H538, 2007.

20. Kleinbongard P, Heusch G and Schulz R: TNFalpha in atherosclerosis, myocardial ischemia/reperfusion and heart failure. Pharmacol Ther 127: 295-314, 2010.

21. Lecour S, Suleman N, Deuchar GA, Somers S, Lacerda L, Huisamen B and Opie LH: Pharmacological preconditioning with tumor necrosis factor-alpha activates signal transducer and activator of transcription-3 at reperfusion without involving classic prosurvival kinases (Akt and extracellular signal-regulated kinase). Circulation 112: 3911-3918, 2005.

22. Kong SS, Liu JJ, Hwang TC, Yu XJ, Lu Y and Zang WJ: Tumour necrosis factor- $\alpha$ and its receptors in the beneficial effects of vagal stimulation after myocardial infarction in rats. Clin Exp Pharmacol Physiol 38: 300-306, 2011. 
23. Omura S, Kawai E, Sato F, Martinez NE, Chaitanya GV Rollyson PA, Cvek U, Trutschl M, Alexander JS and Tsunoda I: Bioinformatics multivariate analysis determined a set of phase-specific biomarker candidates in a novel mouse model for viral myocarditis. Circ Cardiovasc Genet 7: 444-454, 2014.

24. Wu L, Archacki SR, Zhang T and Wang QK: Induction of high STAT1 expression in transgenic mice with LQTS and heart failure. Biochemical and Biophysical Research Communications 358 : 449-454, 2007.

25. Galindo CL, Skinner MA, Errami M, Olson LD, Watson DA, Li J, McCormick JF, McIver LJ, Kumar NM, Pham TQ and Garner HR: Transcriptional profile of isoproterenol-induced cardiomyopathy and comparison to exercise-induced cardiac hypertrophy and human cardiac failure. BMC Physiol 9: 23, 2009.

26. Jie KE, van der Putten K, Wesseling S, Joles JA, Bergevoet MW, Pepers-de Kort F, Doevendans PA, Yasui Y, Liu Q, Verhaar MC, et al: Short-term erythropoietin treatment does not substantially modulate monocyte transcriptomes of patients with combined heart and renal failure. Plos One 7: e41339, 2012.

27. Young ME, Razeghi $\mathrm{P}$ and Taegtmeyer H: Clock genes in the heart: Characterization and attenuation with hypertrophy. Circ Res 88: 1142-1150, 2001.

28. Gréchez-Cassiau A, Rayet B, Guillaumond F, Teboul M and Delaunay F: The circadian clock component BMAL1 is a critical regulator of p21WAF1/CIP1 expression and hepatocyte proliferation. J Biol Chem 283: 4535-4542, 2008.

29. Whitfield JB: Genetic insights into cardiometabolic risk factors Clin Biochem Rev 35: 15-36, 2014.

30. Benderdour M, Fahmi H, Beaudet F, Fernandes JC and Shi Q: Nuclear receptor retinoid-related orphan receptor $\alpha 1$ modulates the metabolic activity of human osteoblasts. J Cell Biochem 112: 2160-2169, 2011

31. Tokudome S, Sano M, Shinmura K, Matsuhashi T, Morizane S, Moriyama H, Tamaki K, Hayashida K, Nakanishi H, Yoshikawa N, et al: Glucocorticoid protects rodent hearts from ischemia/reperfusion injury by activating lipocalin-type prostaglandin D synthase-derived PGD2 biosynthesis. J Clin Invest 119: 1477-1488, 2009.

32. Oakley RH and Cidlowski JA: Glucocorticoid signaling in the heart: A cardiomyocyte perspective. J Steroid Biochem Mol Biol 153: 27-34, 2015

33. Ashton KJ, Tupicoff A, Williams Pritchard G, Kiessling CJ, See Hoe LE, Headrick JP and Peart JN: Unique transcriptional profile of sustained ligand-activated preconditioning in pre- and post-ischemic myocardium. Plos One 8, 2013.
34. Inoue T, Eguchi Y, Matsumoto T, Kijima Y, Kato Y, Ozaki Y, Waseda K, Oda H, Seiki K, Node K and Urade Y: Lipocalin-type prostaglandin D synthase is a powerful biomarker for severity of stable coronary artery disease. Atherosclerosis 201: 385-391, 2008.

35. Miwa Y, Oda H, Shiina Y, Shikata K, Tsushima M, Nakano S, Maruyama T, Kyotani S, Eguchi N, Urade Y, et al: Association of serum lipocalin-type prostaglandin D synthase levels with subclinical atherosclerosis in untreated asymptomatic subjects. Hypertens Res 31: 1931-1939, 2008.

36. Chang DF, Belaguli NS, Iyer D, Roberts WB, Wu SP, Dong XR, Marx JG, Moore MS, Beckerle MC, Majesky MW and Schwartz RJ: Cysteine-rich LIM-only proteins CRP1 and CRP2 are potent smooth muscle differentiation cofactors. Dev Cell 4: 107-118, 2003.

37. Yu TS, Moctezuma-Anaya M, Kubo A, Keller G and Robertson S: The heart LIM protein gene ( Hlp ), expressed in the developing and adult heart, defines a new tissue-specific LIM-only protein family. Mech Dev 116: 187-192, 2002.

38. Zhang L, Hoffman JA and Ruoslahti E: Molecular profiling of heart endothelial cells. Circulation 112: 1601-1611, 2005.

39. Dobaczewski M, Gonzalez Quesada C and Frangogiannis NG: The extracellular matrix as a modulator of the inflammatory and reparative response following myocardial infarction. J Mol Cell Cardiol 48: 504-511, 2010

40. Acharya A, Baek ST, Huang G, Eskiocak B, Goetsch S, Sung CY, Banfi S, Sauer MF, Olsen GS, Duffield JS, et al: The bHLH transcription factor Tcf21 is required for lineage-specific EMT of cardiac fibroblast progenitors. Development 139: 2139-2149, 2012.

41. Conti A, Fabbrini F, D'Agostino P, Negri R, Greco D, Genesio R, D'Armiento M, Olla C, Paladini D, Zannini M and Nitsch L: Altered expression of mitochondrial and extracellular matrix genes in the heart of human fetuses with chromosome 21 trisomy. BMC Genomics 8: 268, 2007

42. Cai XY, Lu L, Wang YN, Jin C, Zhang RY, Zhang Q, Chen QJ and Shen WF: Association of increased S100B, S100A6 and $\mathrm{S} 100 \mathrm{P}$ in serum levels with acute coronary syndrome and also with the severity of myocardial infarction in cardiac tissue of rat models with ischemia-reperfusion injury. Atherosclerosis 217: 536-542, 2011.

This work is licensed under a Creative Commons Attribution-NonCommercial-NoDerivatives 4.0 International (CC BY-NC-ND 4.0) License. 